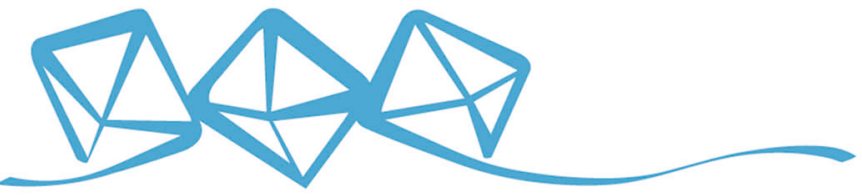 COMMUNICATIONS MATERIALS
}

ARTICLE

https://doi.org/10.1038/s43246-020-0024-3

OPEN

\section{Stable microstructure in a nanocrystalline copper-tantalum alloy during shock loading}

\author{
B. Chad Hornbuckle ${ }^{1 凶}$, Cyril L. Williams ${ }^{1}$, Steven W. Dean ${ }^{1}$, Xuyang Zhou ${ }^{2}$, Chaitanya Kale ${ }^{3}$, Scott A. Turnage ${ }^{1}$, \\ John D. Clayton', Gregory B. Thompson², Anit K. Giri ${ }^{1}$, Kiran N. Solanki (iD ${ }^{3}$ \& Kristopher A. Darling ${ }^{1}$
}

The microstructures of materials typically undergo significant changes during shock loading, causing failure when higher shock pressures are reached. However, preservation of microstructural and mechanical integrity during shock loading are essential in situations such as space travel, nuclear energy, protection systems, extreme geological events, and transportation. Here, we report ex situ shock behavior of a chemically optimized and microstructurally stable, bulk nanocrystalline copper-tantalum alloy that shows a relatively unchanged microstructure or properties when shock compressed up to $15 \mathrm{GPa}$. The absence of shockhardening indicates that the grains and grain boundaries that make up the stabilized nanocrystalline microstructure act as stable sinks, thereby annihilating deformation-induced defects during shock loading. This study helps to advance the possibility of developing advanced structural materials for extreme applications where shock loading occurs.

\footnotetext{
${ }^{1}$ Weapons and Materials Research Directorate, Army Research Laboratory, Aberdeen Proving Ground, MD 21005, USA. ${ }^{2}$ Department of Metallurgical \& Materials Engineering, University of Alabama, Tuscaloosa, AL 35487, USA. ${ }^{3}$ School for Engineering of Matter, Transport, and Energy, Arizona State University, Tempe, AZ 85281, USA. ${ }^{凶}$ email: billy.c.hornbuckle.civ@mail.mil
} 
$\mathrm{M}$ any future applications that aim to harness, avoid, or mitigate the collision of shock waves with condensed matter (and the extreme pressures/temperatures that result) are yet to be realized, primarily due to the lack of new advanced structural materials. Central to this predicament is the discovery of materials, which must preserve their microstructural integrity under intense loading and temperature ${ }^{1-5}$. Therefore, it is imperative to understand factors that alter the sub-features, which control both structural and functional properties, in particularly under conditions of shock physics that represent one of the most extreme environments where virtually every polycrystalline structural materials' microstructure breaks-down (significant changes) ${ }^{1}$. Under shock compression loading, the intense strain rates and driving pressure generated are revealed through the post mortem changes in the residual microstructure. Inevitably, the resultant microstructure consists of high nonequilibrium concentrations of lattice defects such as vacancy clusters, dislocation cells/networks, stacking faults, deformation twins, etc. The generation of these defects is dependent on numerous experimental factors such as peak shock pressure/ stress, pulse duration, strain-rate, temperature, and number of material-dependent parameters. In coarse-grained polycrystalline materials, this rearrangement of the microstructure leads to significant increase in the residual hardness (as much as 100\%) and reduction in the ductility, which can result in failure of the ductile material in a brittle manner ${ }^{2}$.

Within the field of shock physics, the interaction of shock waves with nanocrystalline (NC) metals is a relatively unexplored area of research. Unlike traditional coarse-grained polycrystalline metals, NC metals are exceedingly susceptible to microstructural instabilities due to their far from equilibrium state. This microstructural instability has manifested itself as rapid and extensive grain growth due to all forms of deformation including compression, indentation, tension, wear, fatigue, and creep ${ }^{6-16}$. For instance, room temperature indentation experiments by Zhang et al. ${ }^{6}$ on pure $\mathrm{NC}-\mathrm{Cu}$ resulted in rapid grain coarsening from 20 $\mathrm{nm}$ to about $200-300 \mathrm{~nm}(1000 \%$ increase in the initial mean grain size). See Supplementary Fig. 1 for a practical/stark example of instability in NC-Cu. This instability becomes exacerbated when testing conditions become more and more extreme ${ }^{17}$.

To date, not a single publication exists on the experimental shock testing of bulk NC metals (average grain size $\leq 100 \mathrm{~nm}$ in all three dimensions) and sample size $>5 \mathrm{~mm}$ in 3 dimensions. The lack of publications is due to the difficulties in consolidating NC metals because of their poor thermal microstructural stability as well as overall sample dimensions. Consequently, the vast majority of literature has been devoted to computational modeling efforts of simulated microstructures ${ }^{18-31}$. With regard to the limited experimental studies, the work has utilized laser-driven compression of physically deposited thin films or electrodeposits $(<500 \mu \mathrm{m})^{32}$. Within that subset, the number of laser-based studies presenting post-mortem microstructural characterization is limited to just a few (e.g., ${ }^{33,34}$ ). Specifically, Lu et al., observed $\sim 50 \%$ increase in the post-deformed strength along with rapid grain coarsening from average $70 \mathrm{~nm}$ to $1400 \mathrm{~nm}$ in pure NC $\mathrm{Ta}^{34}$. However, the physics governing the wave propagation and its ability to obtain an equilibrium state in laser-based experiments leaves many questions as to the stress state within the material. An active debate on comparing and drawing definitive conclusions between laser-driven experiments to conventional shock recovery studies is still ongoing.

Recent advances in stabilizing NC alloys are allowing investigation of their true mechanical response, i.e., in the absence of grain growth. This microstructural stabilization has revealed drastic deviations in the expected mechanical response under various extreme conditions $s^{5,35,36}$. To build off this, we are reporting the first comprehensive ex-situ shock experimental behavior of a chemically optimized and microstructurally stable bulk NC copper $(\mathrm{Cu})-3 a t . \%$ tantalum $(\mathrm{Ta})$ material, (designated $\mathrm{NC}-\mathrm{Cu}-3 \mathrm{Ta}$ ). This optimization is in reference to a systematic study that worked through various solute contents to understand their effect on interplay between cluster stability, precipitation hardening effects, and the overall stability of the matrix average grain size. The resilience of this material is demonstrated by relatively unchanged microstructure and the absence of hardening despite being shock compressed up to $15 \mathrm{GPa}$, i.e., this indicates the material's ability to resist/annihilate high defect accumulation. This is the first time such microstructural insensitivity has been observed at these extreme conditions and is in contrary to the findings for any other known alloys.

\section{Results}

Microstructure before shock loading. The material for this study was processed through mechanically alloyed powders using a multi-pass high-temperature equal channel angular extrusion (ECAE) at $700^{\circ} \mathrm{C}$. Complete details of the powder processing and consolidation efforts can be found in ${ }^{5,35}$ and also Supplementary Methods. Primary microstructural analysis of the as-consolidated test samples using transmission electron microscopy (TEM, see Supplementary Methods) indicates that the extruded microstructure for this alloy was found to have an average grain size of $87 \pm 15 \mathrm{~nm}$ with Ta based nanoclusters having an average diameter of $3.2 \pm 0.9 \mathrm{~nm}$, see Supplementary Methods. These Ta based nanoclusters are observed to exist within the $\mathrm{Cu}$ matrix and along grain boundaries. To evaluate microstructural evolution and deformation mechanism under extreme conditions, the $\mathrm{NC}-\mathrm{Cu}-3 \mathrm{Ta}$ disks were shock compressed, released, and recovered following the procedure outlined in Supplementary Methods as well as using the shock recovery assembly shown schematically in Supplementary Fig. 4 for conventional shock recovery. Two samples were shock compressed to $\sim 8$ and $15 \mathrm{GPa}$, respectively under which some plastic deformation occurred, then released back to ambient conditions using a conventional gas gun. Following soft recovery, the specimens were analyzed using TEM and Precession Electron Diffraction (PED) as well as hardness mapping.

Mechanical response after shock-compression loading. The residual structure-property relationships in shock compressed samples were probed through TEM and hardness measurements. Structure-property relationships have been extensively studied for several decades via shock recovery experiments in various coarsegrained materials ${ }^{1,2,37-39}$. In general, these results show that for coarse-grained metals and metallic alloys, the residual hardness exhibits an empirical linear relationship as a function of the square root of peak shock pressure. This relationship holds true up to a critical shock stress beyond which geometric or thermal softening dominates the response ${ }^{1,38}$. This relationship has been generally represented by the following empirical forms $s^{5,14}$ :

$$
\begin{gathered}
\sigma=\sigma_{o}+\alpha \mu b \sqrt{\rho}+K_{1} d^{-1}+K_{2} \Delta^{-1 / 2} \\
\left(\sigma-\sigma_{o}\right)=\zeta\left(H-H_{o}\right)=2 \alpha \mu b \sqrt{P}
\end{gathered}
$$

where $\sigma$ and $\sigma_{o}$ are the post and the pre-shock yield strength, respectively, $\rho$ is the dislocation density, $K_{1}$ and $K_{2}$ are material parameters, $\alpha$ is a constant $\cong 0.5, \mu$ is the shear modulus, $d$ is the dislocation cell size, $\Delta$ is the twin spacing, $b$ is the magnitude of the Burgers vector, $\zeta$ is a constant, $H$ and $H_{o}$ are the post and preshock hardness, and $P$ is the peak shock pressure. Both equations suggest that increasing the peak shock pressure will increase the dislocation density, and consequently, the post-shock yield stress 

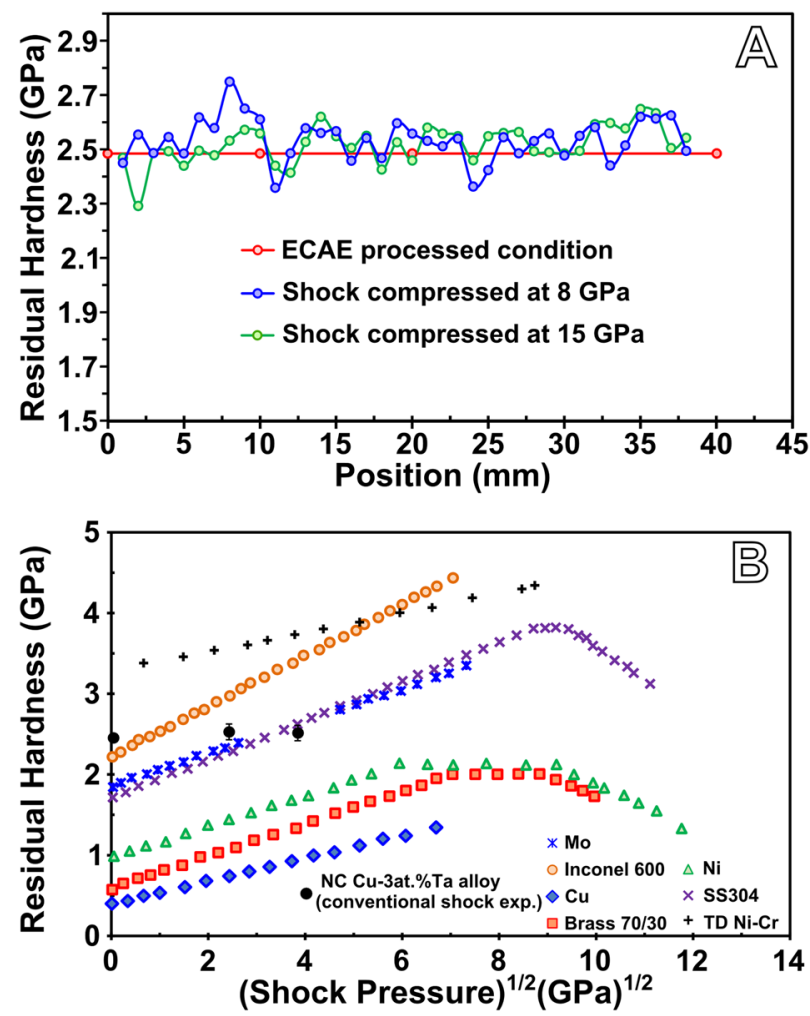

Fig. 1 Post-deformed mechanical behavior of NC-Cu-3Ta and its comparison with advanced structural alloys. a The hardness of the asreceived NC-Cu-3Ta alloy sample compared to the residual hardness of the samples shock compressed to $\sim 8 \mathrm{GPa}$ and $15 \mathrm{GPa}$ using a conventional shock recovery technique, respectively. $\mathbf{b}$ The residual hardness as a

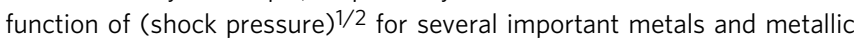
alloys ${ }^{1,38}$

and the residual hardness. Figure 1a shows negligible changes in the residual hardness across the through-thickness direction between the as-received material and samples shock compressed to $\sim 8$ and $15 \mathrm{GPa}$, respectively through conventional shock recovery experiments in $\mathrm{NC}-\mathrm{Cu}-3 \mathrm{Ta}$. Such behavior is anomalous for metals and metallic alloys shock compressed to such high pressures. For instance, as shown in (Fig. 1b), the hardness in pure $\mathrm{Cu}$ and brass is found to increase by factors of two and three, respectively. However, the $\mathrm{NC}-\mathrm{Cu}-3 \mathrm{Ta}$ alloy (i.e., $97 \% \mathrm{Cu}$ ) did not exhibit any significant changes. To the best of the author's knowledge, this type of behavior has never been reported before in the open literature for any structural materials including the work of Bringa et al. ${ }^{40}$, which shows significant increase in material's strength as a function of shock pressure. Whereas, increases in strength of conventional coarse-grained (FCC) materials, i.e., aluminum, copper, and nickel, shocked under similar conditions to those performed in this study are the function of drastic alterations in their microstructure, be it dislocation cells/networks, stacking faults, deformation twins, and vacancy clusters ${ }^{41-44}$. For a cursory review with visual examples of the drastic changes $\mathrm{Cu}$ and other FCC microstructures undergo as a result of shock loading please see reference ${ }^{45}$.

Stable defect density and their interaction with shock compression loading. To address such anomalous strength behavior, we drive our attention to the microstructures of post-shocked samples at various pressure conditions. In general, during shock loading, evidence of plastic deformation is usually revealed in the residual microstructure. The residual microstructure may consist of extremely high concentrations of lattice defects such as vacancy clusters, dislocation cells/networks, stacking faults, deformation twins, etc. ${ }^{45}$. The generation of these defects is dependent on numerous factors such as peak shock pressure/stress, pulse duration, strain-rate, temperature, the crystal structure of the material, grain type and size, alloying elements, and second phase particles, which will lead to significant increases in the postshocked hardness. However, for the bulk NC-Cu-3Ta alloy, as shown in Fig. 2, we observed no significant change in the residual microstructure between the different shock pressures despite undergoing plastic deformation. The bright-field STEM (BFSTEM) images in (Fig. 2a-c) represent the low magnification of the as-received, shock compressed to $\sim 8$ and $15 \mathrm{GPa}$ samples, respectively. Similarly, (Fig. $2 \mathrm{~d}-\mathrm{f}$ ) represents a higher magnification of the as-received and various shock compressed samples. The slight difference in the microstructures of (Fig. $2 \mathrm{~d}$ and e) is the Ta particles pinning the grain boundary in (Fig. 2d) whereas (Fig. 2e) shows a more homogenous distribution of the Ta particles within the grain interior. The TEM images do not reveal significant signs of localized plastic deformation, i.e., dislocation sub-cell formation and or extensive twinning activities, except in the case of the highest shock pressure (e.g., (Fig. 2f)) where dislocations are pinned by nanoclusters. The small dislocation activity does correlate to an insignificant hardness increase in the post-deformed samples (Fig. 1). Generally, through complex modeling, it has been shown that the grain boundaries in NC metals can absorb dislocations and other defects during active deformation $^{46}$. Nevertheless, as mechanical testing becomes more extreme, as in high rate and shock loading; un-stabilized NC metals will undergo drastic coarsening and begin to function as conventional metals by storing high concentrations of lattice defects within the grain interior. In other words, the absence of grain growth and microstructural evolution in NC-Cu-3Ta signals that the stabilized grains and grain boundaries act as stable sinks and persist, thereby annihilating deformation-induced defects during shock compression loadings. Further, no cracking or voids were observed at the grain boundaries or $\mathrm{Cu}-\mathrm{Ta}$ interfaces. Overall, the microstructural characterization of NC-Cu-3Ta shown in Fig. 2, supports the earlier observation of negligible post-shocked residual hardness increase despite undergoing plastic deformation (see Supplementary Fig. 5 for shock wave profiles confirming elastic-plastic wave structure).

To evaluate the influence of the extreme pressure jump on the textural changes (grain boundary sliding and grain rotation processes), precession electron diffraction measurements were performed in various regions. The orientation-imaging map (OIM) in Fig. 3 shows a high degree of randomness in the orientation relationships between the $\mathrm{Cu}$ grains in the as-received state and the fully released states with a similar texture index (see Supplementary Methods for Supplementary Figs. 2 and 3 for grain boundary character distributions and pole figures). In addition, only a marginal change in the average grain size was observed between the as-received sample and the shock recovered samples. This is true even for the $\sim 15 \mathrm{GPa}$ sample where the vast majority of the microstructure remains relatively un-coarsened. An important takeaway from these results is that although there may be a small change in the average grain size; however, no significant changes in the materials hardness value were observed. In our previous study 47 , it was found that the dislocationnanocluster strength obtained from atomistic simulations at $298 \mathrm{~K}$ amounts to a considerable portion of the experimental flow strength as compared to average grain size. Thus, the observations made on recovered shock compressed $\mathrm{NC}-\mathrm{Cu}-3 \mathrm{Ta}$ samples clearly indicate the important role of $\mathrm{Ta}$ nanoclusters on the structural stability as well as strength of these alloys. These results also allude to the fact that the small change to average grain size 

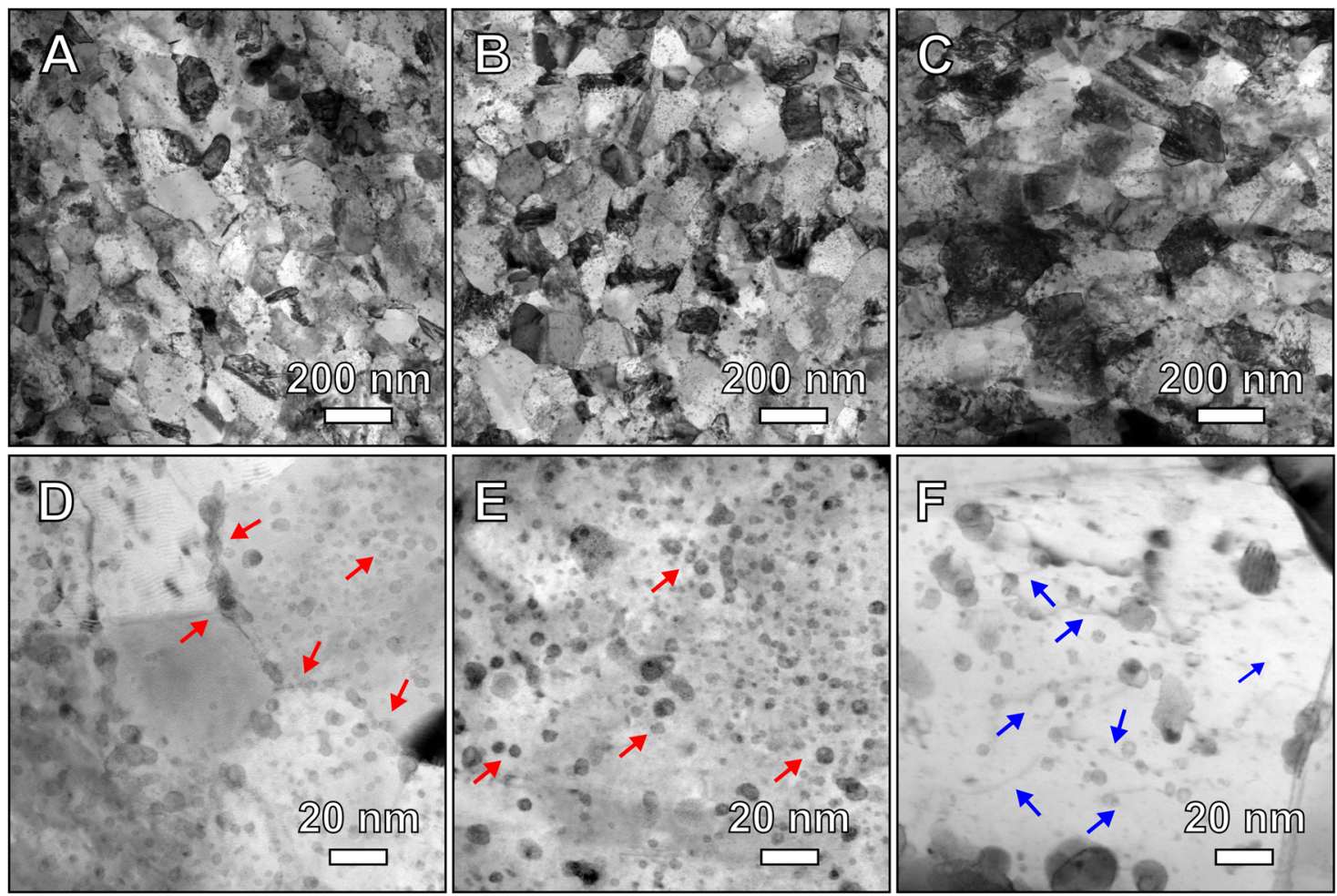

Fig. 2 The bright-field STEM (BF-STEM) characterization of microstructural evolutions acquired for as-received and shock compressed bulk

NC-Cu-3Ta alloy. The BF-STEM images a-c represent the low magnification of the as-received, shock compressed to $\sim 8$ and 15 GPa samples, respectively. Similarly, $\mathbf{d}-\mathbf{f}$ represents a higher magnification of the as-received and various shock compressed samples. Red arrows indicate Ta nanoclusters and the blue arrows highlights dislocation interaction with the Ta nanocluster.
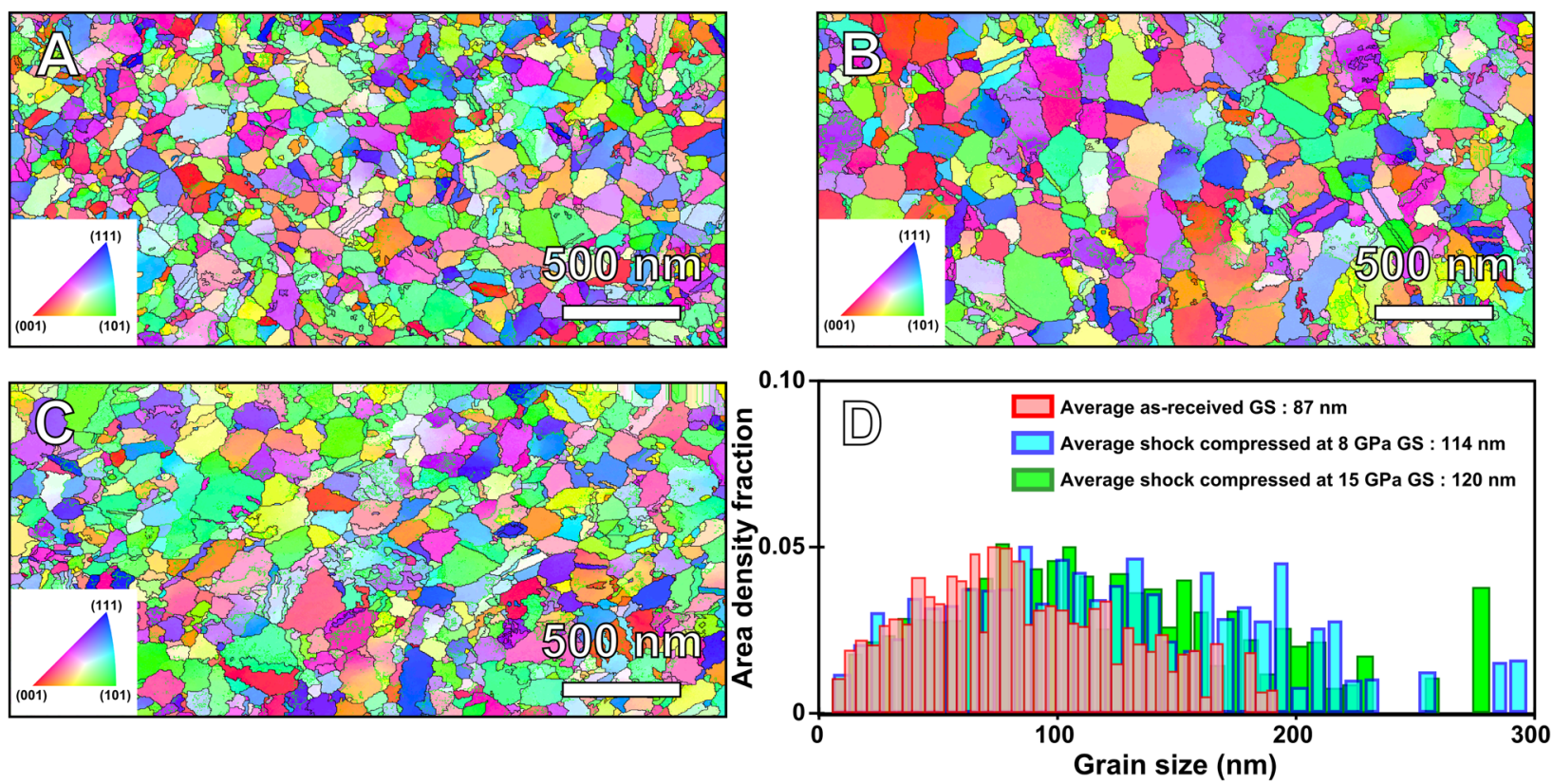

Fig. 3 Microstructural data acquired for as-received and shock compressed bulk NC-Cu-3at.\% Ta alloy using Precession Electron Diffraction (PED) microscopy. Precession electron diffraction image of (a) as-received material, (b) material shock compressed to $\sim 8 \mathrm{GPa}$, and (c) material shock compressed to $\sim 15 \mathrm{GPa}$. d The distributions indicate nominal increase in $\mathrm{Cu}$ grain size with the majority of the grains remaining in the $\mathrm{NC}$ regime.

following shock compression and release is indeed negligible. Therefore, the overall lack of microstructure and texture evolution in this bulk NC-Cu-3Ta alloy is atypical for metals and metallic alloys shock compressed to $\sim 15 \mathrm{GPa}$ and is deemed anomalous ${ }^{45}$. This is especially true for conventional NC metals (i.e., un-stabilized) where microstructures are readily susceptible to perturbations in environmental conditions than their coarsergrained counterparts ${ }^{6-16}$. In fact, $\mathrm{NC}$ pure $\mathrm{Cu}$ is an extreme 
example of this, whereby its microstructure is so unstable that it is known to coarsen over time even under ambient laboratory temperature/pressure, and under deformation, this instability is extremely exacerbated ${ }^{48}$. See Supplementary Fig. 1 for an example of just how unstable nanocrystalline $\mathrm{Cu}$ can be as a result of deformation.

\section{Discussion}

We analyzed the plastic deformation mechanisms active in $\mathrm{NC}-\mathrm{Cu}-\mathrm{Ta}$ alloy during shock compression, i.e., the role of dislocation nucleation and propagation velocity as well as twining and the relaxation processes, which result from these mechanisms. For moderate to high shock stresses, such as those reported in this letter, plastic deformation is governed by the mobile dislocation density and the average mobile dislocation velocity (approaching the shear wave velocity) through the classical Orowan relationship $(\dot{\varepsilon}=b \rho \nu$, where $\dot{\varepsilon}$ is the applied strain rate, b is the Burgers vector, $\rho$ is the dislocation density, and $v$ is the dislocation velocity). However, using atomistic simulations, it was recently predicted that $\mathrm{NC}-\mathrm{Cu}-\mathrm{Ta}$ alloys exhibit a reduced dislocation gliding velocity stemming from the stability of the controlling microstructural length scale such as the grain size and Ta nanocluster size/spacing, which act as barriers that pin/slow down defect propagation ${ }^{35}$. In addition, it was also shown that the introduction of $\mathrm{Ta}$ clusters as well as the increased grain boundary volume results in an increased phonon density of states such that phonon-drag forces would play a larger role on mobile dislocations in $\mathrm{NC}-\mathrm{Cu}-\mathrm{Ta}$ alloys through an increased drag coefficient $^{35}$. Based on the Orowan equation, both of these factors indicate that the mobile dislocation density in $\mathrm{Cu}-\mathrm{Ta}$ alloys must be much higher than observed. In fact, extremely high nonequilibrium concentrations of defects have been routinely observed post mortem in conventional coarse-grained $\mathrm{Cu}$ and many other alloys after shock testing under similar conditions reported here ${ }^{1,38}$. The lack of accumulated defect structures such as the formation of cell structures (indicative of high dislocation density) within the microstructure of $\mathrm{NC}-\mathrm{Cu}-\mathrm{Ta}$ alloys is then a critical observation.

In regards to twinning, under shock conditions in FCC metals with high stacking fault free energy (SFE) such as pure $\mathrm{Cu}$, twinning threshold stresses decrease with increasing strain rates, i.e., in most, highly shocked $\mathrm{Cu}$ samples twinning has been reported as one of the most common deformation modes ${ }^{37}$. The minimum twinning pressure for coarse-grained $\mathrm{Cu}$ and $\mathrm{Cu}$ alloys is in the range of $14-20 \mathrm{GPa}^{37}$. In the case of $\mathrm{NC}-\mathrm{Cu}-3 \mathrm{Ta}$ alloy, an active competition between dislocation motion and twinning is expected to decide which will be the more operative deformation mechanism at high-rates ${ }^{49}$. Nevertheless, the twinning deformation was relatively inactive in $\mathrm{NC}-\mathrm{Cu}-3 \mathrm{Ta}$, indicative of grain size effects on the twin pressure. Further, based on the analyses of the residual microstructure of $\mathrm{NC}-\mathrm{Cu}-3 \mathrm{Ta}$, it is apparent that no increase in dislocation density and mechanical twinning are observed, i.e., stabilized NC alloys likely exhibit an ability to resist high defect (such as dislocation) accumulation and damage. In contrast, most polycrystalline $\mathrm{Cu}$ shocked at as low as $5 \mathrm{GPa}$ show significant increase in the dislocation density as well as postdeformed residual hardness ${ }^{45}$. Therefore, such resistance in $\mathrm{NC}-\mathrm{Cu}-3 \mathrm{Ta}$ has not been observed before and is analogous to the ability of NC metals to absorb radiation damage, where stable interfaces act as potent sinks for point defects. Here in the same way, the thermo-mechanically stabilized grain boundaries and cluster interfaces persist and continue to operate as sinks to absorb the deformation defects such as dislocations. Such inherent behavior or properties are unlikely to be observed in unstabilized NC metals, where their microstructures would become unstable under the drastically intensified driving force, i.e., as the microstructure evolves the sink density reduces ${ }^{50}$. Therefore, the resilient phenomena of absorbing defects such as dislocations and resisting microstructural evolution under extreme dynamic conditions, as reported here, is only possible in thermo-mechanically stabilized nanocrystalline metals such as $\mathrm{NC}-\mathrm{Cu}-3 \mathrm{Ta}$. In general, the accumulation of defects during shock compression can lead to potential void nucleation sites such as vacancies and vacancy clusters $^{50}$, which can be detrimental to spall failure. Therefore, the ability to maintain a lower defect concentration under shock compression provides an archetype from which materials can be developed that go against conventional theory. That is, smaller grain sizes may then prove to yield higher spall strengths as compared to coarser-grained alloys; this work is ongoing.

In summary, optimized $\mathrm{NC}-\mathrm{Cu}-3 \mathrm{Ta}$ shows extraordinary microstructural stability under extreme loading conditions. Mechanical hardness and TEM results provide evidence of an ability to resist high defect accumulation and damage as compared to other known materials reported in the open literature. This is attributed to the stabilized grains and grain boundaries that act as stable sinks, analogous to NC metals known ability to absorb radiation damage, thereby providing a mechanism of resistance, neutralizing further deformation-induced defects. In other words, this letter highlights an important material science discovery that if a material's microstructure is preserved to the length scale of nanometers through certain stabilization mechanisms, then the material's behavior will be anomalous in nature. These results indicate that stabilized NC materials provide a new frontier for fundamental discovery where opportunities exist that go beyond the perceived mechanical and functional limits of NC metals.

\section{Data availability}

The authors declare that the data supporting the findings of this study are available within the paper [and its Supplementary Information Files].

Received: 17 October 2019; Accepted: 30 March 2020;

Published online: 05 May 2020

\section{References}

1. Meyers, M. A. Dynamic behavior of materials (John wiley \& sons, 1994).

2. Murr, L. E. Residual microstructure-mechanical property relationships in shock-loaded metals and alloys. in Shock waves and high-strain-rate phenomena in metals, 607-673 (Springer, Boston, MA, 1981).

3. Anderson, P. M et al. Mechanical properties of nanostructured metals. In Handbook of nanomaterials properties, 495-553 (Springer, Berlin, Heidelberg, 2014).

4. Ackland, G. Controlling radiation damage. Science 327, 1587-1588 (2010)

5. Darling, K. A. et al. Extreme creep resistance in a microstructurally stable nanocrystalline alloy. Nature 537, 378-381 (2016).

6. Zhang, Kai, Weertman, J. R. \& Eastman, J. A. The influence of time, temperature, and grain size on indentation creep in high-purity nanocrystalline and ultrafine grain copper. Appl. Phys. Lett. 85, 5197-5199 (2004).

7. Zhang, Kai, Weertman, J. R. \& Eastman, J. A. Rapid stress-driven grain coarsening in nanocrystalline $\mathrm{Cu}$ at ambient and cryogenic temperatures. Appl. Phys. Lett. 87, 061921 (2005).

8. Brandstetter, S. et al. Grain coarsening during compression of bulk nanocrystalline nickel and copper. Scripta Mater. 58, 61-64 (2008).

9. Gianola, D. S. et al. Stress-assisted discontinuous grain growth and its effect on the deformation behavior of nanocrystalline aluminum thin films. Acta Materialia 54, 2253-2263 (2006).

10. Rupert, T. J. et al. Experimental observations of stress-driven grain boundary migration. Science 326, 1686-1690 (2009).

11. Sharon, J. A. et al. Stress-driven grain growth in nanocrystalline Pt thin films. Scripta Mater. 64, 25-28 (2011).

12. Fan, G. J. et al. Uniaxial tensile plastic deformation and grain growth of bulk nanocrystalline alloys. Acta Mater. 54, 4781-4792 (2006).

13. Rupert, T. J. \& Christopher, A. S. Sliding wear of nanocrystalline Ni-W: structural evolution and the apparent breakdown of Archard scaling. Acta Mater. 58, 4137-4148 (2010). 
14. Padilla, H. A. \& Boyce, B. L. A review of fatigue behavior in nanocrystalline metals. Exper. Mech. 50, 5-23 (2010).

15. Choi, I.-C. et al. Nanoscale room temperature creep of nanocrystalline nickel pillars at low stresses. Int. J. Plast. 41, 53-64 (2013).

16. Dalla Torre, F., Van Swygenhoven, H. \& Victoria, M. Nanocrystalline electrodeposited Ni: microstructure and tensile properties. Acta Mater. 50, 3957-3970 (2002).

17. Meyers, M. A. et al. Laser compression of nanocrystalline metals. in AIP Conference Proceedings, Vol. 1195. No. 1. (American Institute of Physics, 2009).

18. Chen, J., Tschopp, M. A. \& Dongare, A. M. Shock wave propagation and spall failure of nanocrystalline $\mathrm{Cu} / \mathrm{Ta}$ alloys: effect of $\mathrm{Ta}$ in solid-solution. J. Appl. Phys. 122, 225901 (2017).

19. Hahn, E. N. et al. On the ultimate tensile strength of tantalum. Acta Mater 126, 313-328 (2017)

20. Borodin, E. N. \& Mayer, A. E. Theoretical interpretation of abnormal ultrafine-grained material deformation dynamics. Modell. Simul. Mater. Sci. Eng. 24, 025013 (2016).

21. Mackenchery, K. et al. Dislocation evolution and peak spall strengths in single crystal and nanocrystalline Cu. J. Appl. Phys. 119, 044301 (2016).

22. Liao, Y. I. et al. Molecular dynamics studies of the roles of microstructure and thermal effects in spallation of aluminum. Mech. Mater. 84, 12-27 (2015).

23. Xiang, $\mathrm{M} ., \mathrm{Hu}, \mathrm{H}$. \& Chen, J. Spalling and melting in nanocrystalline $\mathrm{Pb}$ under shock loading: molecular dynamics studies. J. Appl. Phys. 113, 144312 (2013).

24. Ma, W., Zhu, W. \& Hou, Y. A comparative study on shock compression of nanocrystalline $\mathrm{Al}$ and $\mathrm{Cu}$ : shock profiles and microscopic views of plasticity. J. Appl. Phys. 114, 163504 (2013).

25. Yan-Wen, Ma. WenLu Molecular dynamics investigation of shock front in nanocrystalline copper. Acta Phys. Sinica 3, 39 (2013).

26. Dongare, A. M. et al. Atomic scale studies of spall behavior in nanocrystalline Cu. J. Appl. Phys. 108, 113518 (2010).

27. Wang, F. et al. Nanoscale interface of metals for withstanding momentary shocks of compression. Nanoscale 2, 2818-2825 (2010).

28. Ma, Wen, Zhu, W. \& Jing, F. The shock-front structure of nanocrystalline aluminum. Appl. Phys. Lett. 97, 121903 (2010).

29. Jérusalem, A. \& Radovitzky, R. A continuum model of nanocrystalline metals under shock loading. Modell. Simul. Mater. Sci. Eng. 17, 025001 (2008).

30. Jarmakani, H. N. et al. Molecular dynamics simulations of shock compression of nickel: from monocrystals to nanocrystals. Acta Mater. 56, 5584-5604 (2008).

31. Chen, Jie et al. Unraveling the role of interfaces on the spall failure of $\mathrm{Cu} / \mathrm{Ta}$ multilayered systems. Sci. Rep. 10, 1-15. (2020).

32. Remington, T. P. et al. Spall strength dependence on grain size and strain rate in tantalum. Acta Mater. 158, 313-329 (2018).

33. Wang, Y. M. et al. Deforming nanocrystalline nickel at ultrahigh strain rates. Appl. Phys. Lett. 88, 061917 (2006).

34. Lu, C.-H. et al. Laser compression of nanocrystalline tantalum. Acta Mater. 61, 7767-7780 (2013).

35. Turnage, $\mathrm{S}$. A. et al. Anomalous mechanical behavior of nanocrystalline binary alloys under extreme conditions. Nat. Commun. 9, 1-10 (2018).

36. Hornbuckle, B. C. et al. Revealing cryogenic mechanical behavior and mechanisms in a microstructurally-stable, immiscible nanocrystalline alloy. Scripta Mater 160, 33-38 (2019).

37. Sanchez, J. C., Murr, L. E. \& Staudhammer, K. P. Effect of grain size and pressure on twinning and microbanding in oblique shock loading of copper rods. Acta Mater. 45, 3223-3235 (1997).

38. Murr, L. E. Effects of peak pressure, pulse duration, and repeated loading on the residual structure and properties of shock deformed metals and alloys. in Shock waves and high-strain-rate phenomena in metals, 753-777 (Springer, Boston, MA, 1981).

39. Gray, G. T. Influence of shock-wave deformation on the structure/property behavior of materials. in High-pressure shock compression of solids, 187-215 (Springer, New York, NY, 1993).

40. Bringa, E. M. et al. Ultrahigh strength in nanocrystalline materials under shock loading. Science 309, 1838-1841 (2005).

41. Williams, C. L. et al. On the shock stress, substructure evolution, and spall response of commercially pure 1100-O aluminum. Mater. Sci. Eng. A 618, 596-604 (2014).

42. Higgins, D. L. et al. Contrasting the microstructural and mechanical response to shock loading of cold-rolled copper with annealed copper. Metall. Mater. Trans. A 46, 4518-4521 (2015).
43. Follansbee, P. S. \& Gray G. T. Advances in Plasticity. 385 (1989).

44. Esquivel, E. V. et al. Comparison of microstructures for plane shock-loaded and impact crater-related nickel: the microtwin-microband transition. J. Mater. Sci. 38, 2223-2231 (2003).

45. Williams, C. L. 4.2.1 substructure in shock compressed metals with FCC lattice structure. Synth. SEM Lect. Exper. Mech. 2, 60-69 (2019).

46. Van Swygenhoven, H., Derlet, P. M. \& Frøseth, A. G. Nucleation and propagation of dislocations in nanocrystalline fcc metals. Acta Mater. 54, 1975-1983 (2006).

47. Kale, C. et al. Thermo-mechanical strengthening mechanisms in a stable nanocrystalline binary alloy-A combined experimental and modeling study. Mater. Des. 163, 107551 (2019).

48. Gai, P. L., Zhang, K. \& Weertman, J. Electron microscopy study of nanocrystalline copper deformed by a microhardness indenter. Scripta Mater. 56, 25-28 (2007).

49. Bhatia, M. A. et al. The role of $\mathrm{Ta}$ on twinnability in nanocrystalline $\mathrm{Cu}-\mathrm{Ta}$ alloys. Mater. Res. Lett. 5, 48-54 (2017).

50. Kressel, H. \& Brown, N. Lattice defects in shock-deformed and cold-worked nickel. J. Appl. Phys. 38 1618-1625 (1967).

\section{Acknowledgements}

The authors acknowledge: A.J. Roberts and T.L. Luckenbaugh for synthesis of the $\mathrm{Cu}-\mathrm{Ta}$ powder; T.L. Luckenbaugh, S. Marsh, and M.C. Aniska for consolidation of the $\mathrm{Cu}-\mathrm{Ta}$ powder; S. Marsh and M.C. Aniska for machining of $\mathrm{Cu}-\mathrm{Ta}$ test specimen; and Dr. R.C. Becker and Dr. S. Satapathy for fruitful discussion. C.K. and K.N.S. acknowledge the support of US Army Research Laboratory and the National Science Foundation under contracts W911NF-15-2-0038 and 1663287. X.Z. and G.B.T. gratefully acknowledge the NSF-DMR-1709803 for support.

\section{Author contributions}

K.A.D., C.L.W., A.K.G., and K.N.S. equally contributed to developing the idea. K.A.D., B.C.H., and A.K.G processed the nanocrystalline materials. C.L.W. and S.A.T. performed the shock recovery experiments. B.C.H., X.Z., and G.B.T. performed the microstructural characterization. K.A.D., K.N.S., B.C.H., C.L.W., S.D., and C.K., analyzed the data. K.A.D., K.N.S., C.L.W., B.C.H., J.D.C., and S.D. wrote the paper. K.A.D., B.C.H., X.Z., and C.K. edited the figures. K.N.S. supervised C.K., and G.B.T. supervised X.Z.

\section{Competing interests}

The authors declare no competing interests.

\section{Additional information}

Supplementary information is available for this paper at https://doi.org/10.1038/s43246 020-0024-3.

Correspondence and requests for materials should be addressed to B.C.H.

Reprints and permission information is available at http://www.nature.com/reprints

Publisher's note Springer Nature remains neutral with regard to jurisdictional claims in published maps and institutional affiliations.

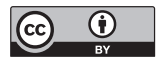

Open Access This article is licensed under a Creative Commons Attribution 4.0 International License, which permits use, sharing, adaptation, distribution and reproduction in any medium or format, as long as you give appropriate credit to the original author(s) and the source, provide a link to the Creative Commons license, and indicate if changes were made. The images or other third party material in this article are included in the article's Creative Commons license, unless indicated otherwise in a credit line to the material. If material is not included in the article's Creative Commons license and your intended use is not permitted by statutory regulation or exceeds the permitted use, you will need to obtain permission directly from the copyright holder. To view a copy of this license, visit http://creativecommons.org/ licenses/by/4.0/.

This is a U.S. government work and not under copyright protection in the U.S.; foreign copyright protection may apply 2020 